\title{
Structural Impact of Three Parkinsonism-Associated Missense Mutations on \\ Human DJ-1
}

Mahadevan Lakshminarasimhan ${ }^{+\dagger}$, Marien T. Maldonado ${ }^{+\dagger}$, Wenbo Zhou ${ }^{\#}$, Anthony L. Fink $^{\#}$, and Mark A. Wilson ${ }^{+*}$

${ }^{+}$Department of Biochemistry and the Redox Biology Center, The University of NebraskaLincoln, Lincoln, NE, 68588-0664 and ${ }^{\#}$ Department of Chemistry and Biochemistry, The University of California Santa Cruz, CA, 95064

${ }^{\dagger}$ Both authors contributed equally to this work

${ }^{*}$ To whom correspondence should be addressed

\section{SUPPORTING INFORMATION}

\begin{tabular}{lll}
\hline Protein & $\begin{array}{l}\text { Dimeric molecular weight } \\
\text { (calculated; Da) }\end{array}$ & $\begin{array}{l}\text { Dimeric molecular weight } \\
\text { (fit; Da) }\end{array}$ \\
\hline Wild-type & 40345 & 37793 \\
A104T & 40405 & 35264 \\
E163K & 40343 & 40876 \\
M26I & 40309 & 36407 \\
\hline
\end{tabular}

Table S1. Comparison of theoretical and fit molecular weights for dimeric DJ-1

determined from the representative sedimentation equilibrium ultracentrifugation

experiment illustrated in Figure 3. The theoretical molecular weights are those calculated for the tag-cleaved protein (see Experimental Procedures), which includes a GSH- "scar" sequence at the $\mathrm{N}$-terminus of each protein resulting from thrombin cleavage of the $\mathrm{N}$ terminal hexa-histidine tag. 


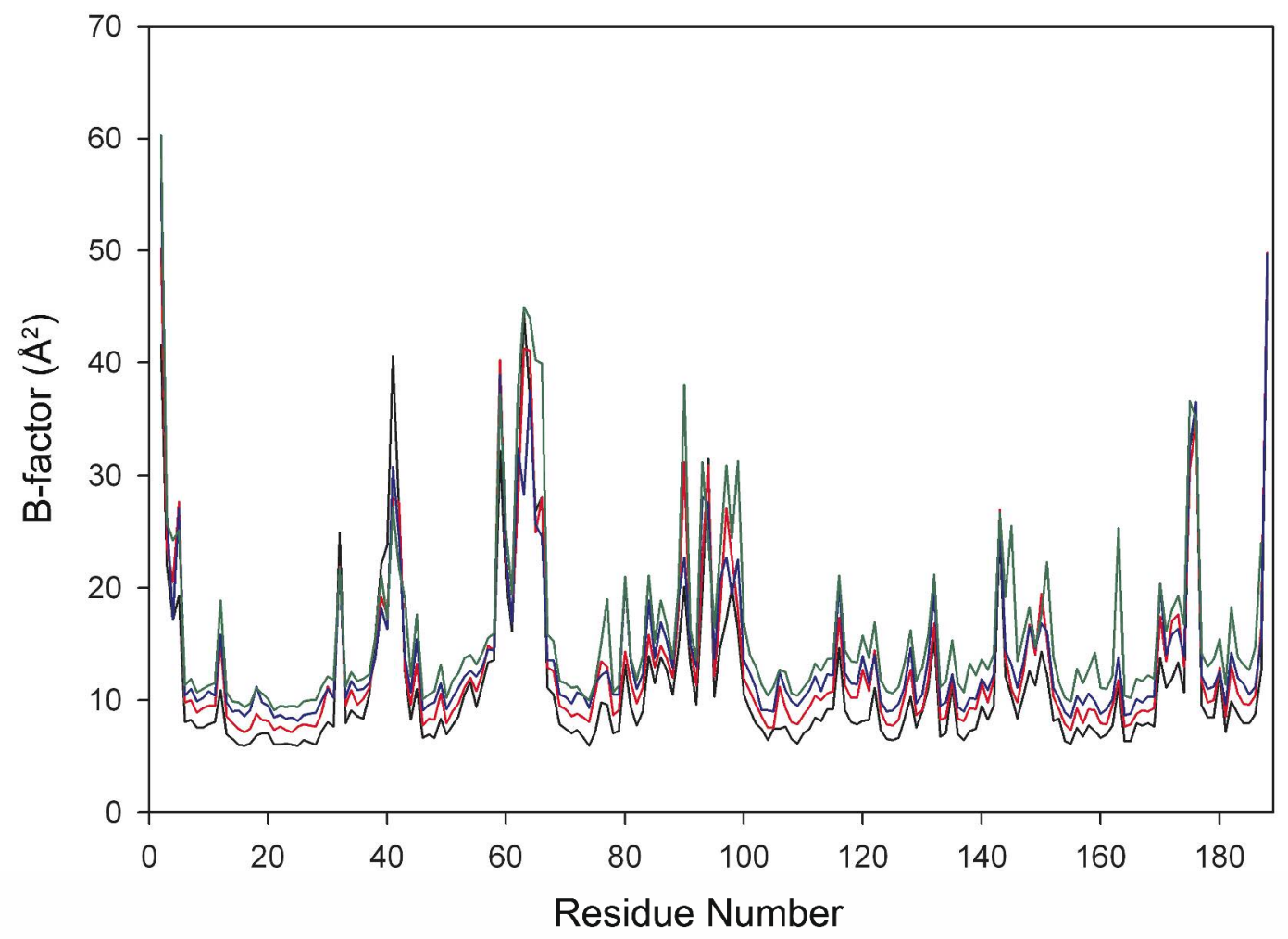

Figure S2. Comparison of refined $B_{\mathrm{eq}}$ factors for DJ-1. The residue averaged B-factors for wild-type (PDB 1SOA; black), M26I (red), A104T (blue) and E163K (green) DJ-1 are shown as a function of residue number. Each structure was refined in SHELX-97 with anisotropic displacement parameters; therefore the $\mathrm{B}_{\mathrm{eq}}$ is the isotropic equivalent $\mathrm{B}$ factor as reported in the PDB. Overall, the four proteins display very similar distributions of B-factors, with some noticeable differences for E163K from residues 140-180.

Interestingly, the average B-factor for each protein is inversely correlated with its $\mathrm{T}_{\mathrm{m}}$ (see Table 1 and Figure 2), as demonstrated above by the B-factor baseline offsets that increase as measured stability of the protein decreases (rank order of stability: wt $>$ M26I $>$ A104T $>$ E163K). We note that many experimental factors can influence the overall B-factor, however, and this correlation may be coincidental. 


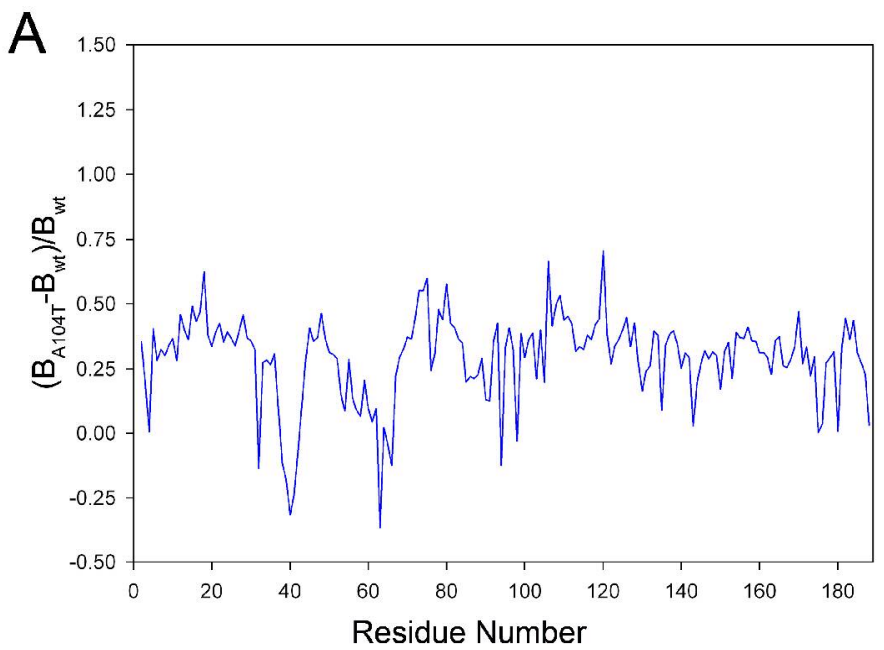

B

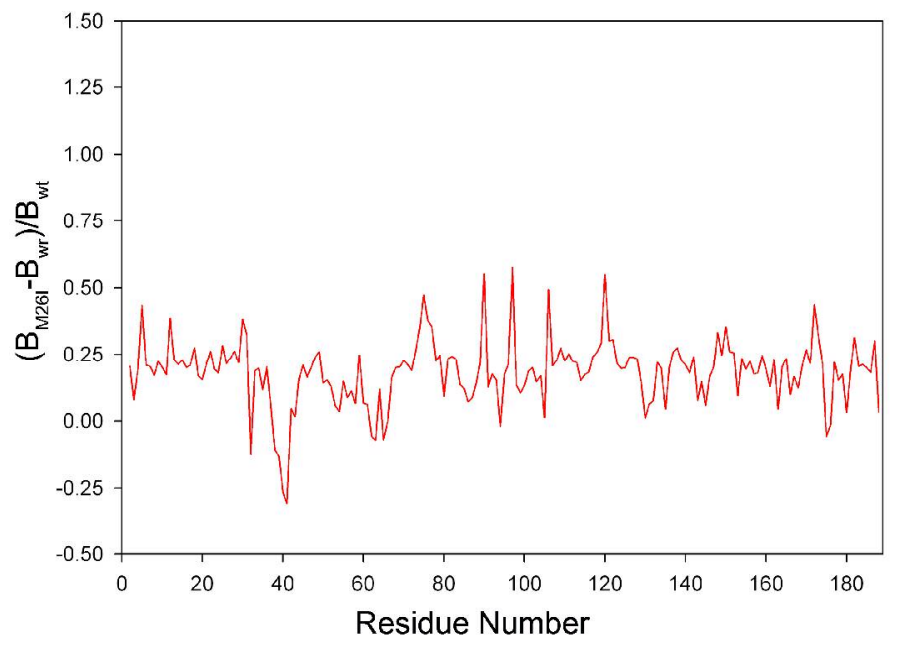

C

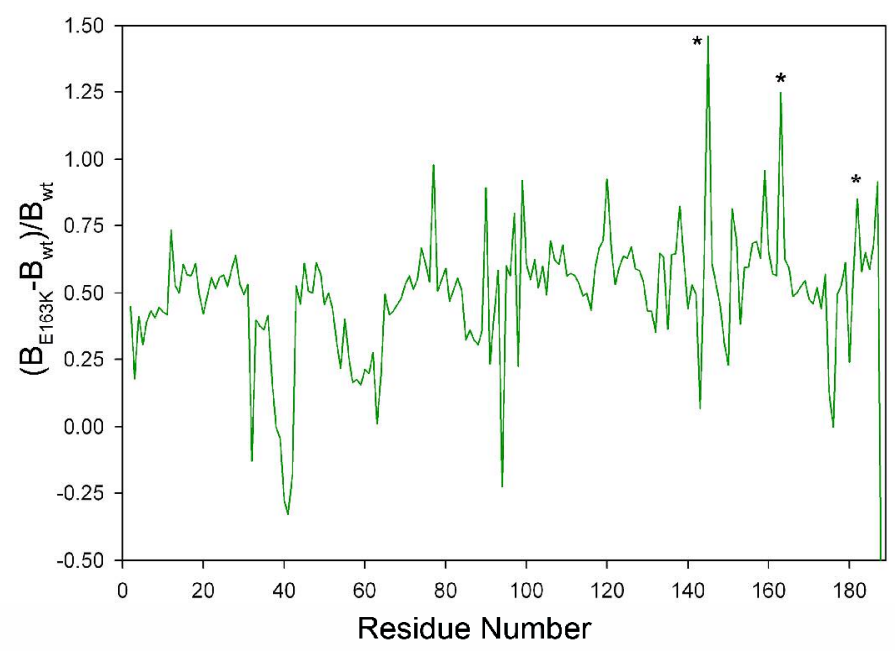


Figure S3. Normalized difference B-factors for each of the three PD-associated DJ-1 mutations. In each panel, the difference between the residue averaged B-factor of the mutant protein and wtDJ-1 (PDB 1SOA) is divided by the residue averaged B-factor of the wild-type protein $\left(\frac{\left\{B_{m u t}-B_{w t}\right\}}{B_{w t}}\right)$ to provide a residue averaged difference B-factor that is weighted by the B-factors of the wild-type protein. This is an approximate form of error scaling, since larger B-factors in the wild-type protein will be associated with larger errors, and the corresponding normalized difference B-factor will be smaller. Panel A shows A104T DJ-1 (blue), Panel B shows M26I DJ-1 (red) and Panel C shows E163K DJ-1 (green). All three mutants show lower mobility than wtDJ-1 in two regions centered around residues 40 and 60. The most significant elevations in normalized difference B-factors are seen in E163K (Panel C), where residues 145, 163 and 186 (indicated with asterisks) are significantly more mobile in the mutant protein. These residues are involved in a dimer-spanning network of hydrogen bonds that is disrupted by the E163K substitution (Figure 7). 